\title{
A Large Mass Occupying the Entire Left Atrium
}

\author{
Masaki Ikeuchi, Kumi Hisano, Yoshiya Monden and Yoshitoshi Urabe
}

Key words: echocardiography, magnetic resonance imaging, heart failure, cerebrovascular disorders, lung neoplasms, thrombus

(Intern Med 52: 2283-2284, 2013)

(DOI: 10.2169/internalmedicine.52.0843)

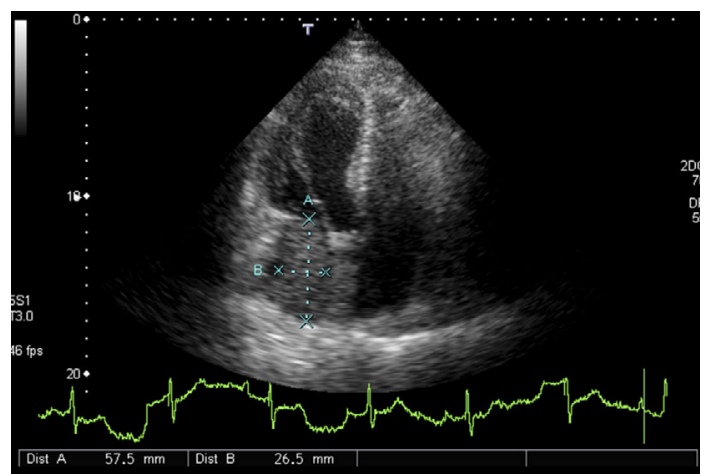

Picture 1.

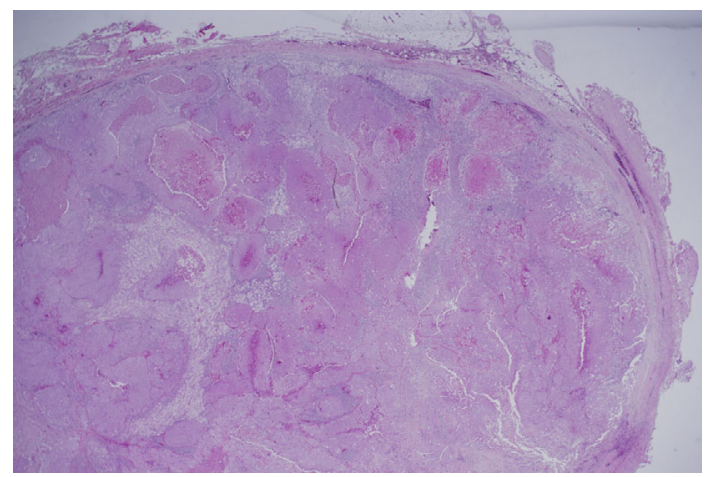

Picture 3.

A 67-year-old man was admitted with sudden onset of right-sided hemiparesis as well as progressive shortness of breath. On admission, diffusion-weighed MRI showed multiple acute brain infarctions. When intracardiac sources of embolism were investigated, transthoracic echocardiography revealed a large, low-echoic and poorly mobile mass that occupied the entire left atrium (LA; Picture 1). Contrastenhanced CT showed that the mass had occluded the right lower pulmonary vein (Picture 2) and revealed multiple

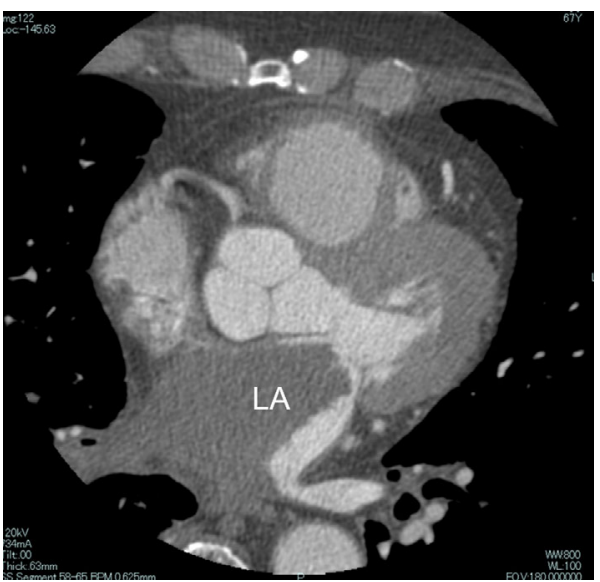

Picture 2.

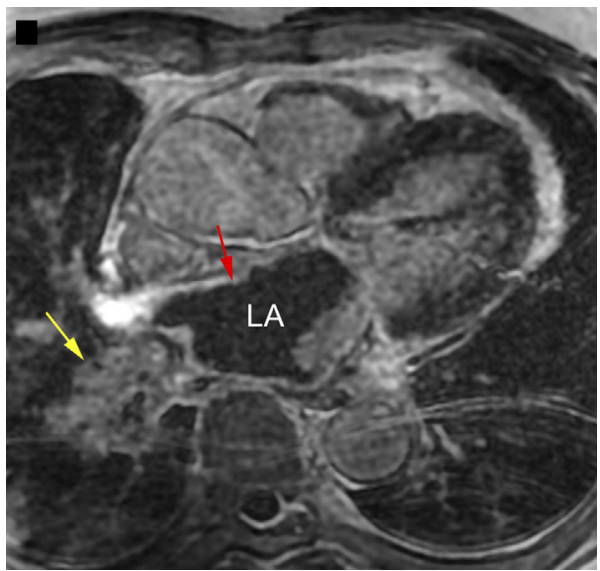

Picture 4.

swollen lymph nodes in the neck and chest. A cervical lymph node biopsy led to a histopathological diagnosis of squamous cell carcinoma (Picture 3). Gadolinium-enhanced chest MRI demonstrated a gray area in the hilar region of the right lower lobe, indicating a vascular tumor (Picture 4, 
yellow arrow), whereas most of the mass in the LA was black and consistent with a thrombus (Picture 4, red arrow). Additional upper gastrointestinal endoscopy detected no esophageal malignancies. These findings strongly suggested that the LA mass was a giant thrombus that developed secondary to the advanced hilar-type squamous cell carcinoma in the lungs. Despite the administration of appropriate anticoagulant therapy, repeat echocardiography confirmed the absence of changes in the size of the mass. The patient died 10 days after admission.

The authors state that they have no Conflict of Interest (COI).

(C) 2013 The Japanese Society of Internal Medicine http://www.naika.or.jp/imonline/index.html 